\title{
VIVÊNCIAS ACADÉMICAS, COMPETÊNCIAS PESSOAIS E SAÚDE MENTAL EM ESTUDANTES DE CIÊNCIAS DA SAÚDE
}

\author{
Rui Porta-Nova ${ }^{1}$ \\ Manuela Fleming 2
}

Resumo: O objectivo deste estudo é identificar as Vivências Académicas que têm maior impacto na Saúde Mental de jovens adultos estudantes do ensino superior na área das Ciências da Saúde e quais as dimensões das Competências Pessoais mais importantes que possam contribuir para a ausência de perturbação psicológica. A investigação incidiu sobre uma amostra de 620 indivíduos, estudantes de Medicina, Enfermagem, Fisioterapia, Cardiopneumologia e Radiologia, com idade média de 20,3 anos, variando entre os 18 e os 25 anos de idade, sendo $81 \%$ do sexo feminino. Os instrumentos utilizados foram um Questionário Demográfico; o Questionário de Vivências Académicas - QVA (Almeida \& Ferreira, 1997, 1999; Ferreira \& Almeida, 1997); o Self-Perception Profile for College Students SPPCS (Neemann \& Harter, 1986; Pais-Ribeiro 1994) e o Mental Health Inventory - MHI (Pais-Ribeiro, 2001; Veit \& Ware, 1983). Foram definidos dois modelos preditores da Saúde Mental para esta amostra. O primeiro modelo, que tem as Vivências Académicas como preditoras, é composto pelas variáveis Bem-Estar Psicológico, Auto-Confiança, Bem-Estar Físico e Percepção Pessoal de Competências Cognitivas. O segundo modelo, com as dimensões das Competências Pessoais como preditoras, contém as variáveis Apreciação Global, Competência Intelectual, Relações com os Pais, Aparência e Relações Amorosas. Discute-se a pertinência dos resultados obtidos e as suas implicações, nomeadamente a importância do Bem-Estar Psicológico e da Auto-Estima, como factores promotores da saúde mental em estudantes dos cursos de Ciências da Saúde.

Palavras-chave: estudantes do ensino superior, ciências da saúde, vivências académicas, competências pessoais, saúde mental.

Academic life experiences, personal competences and mental health in health sciences students (Abstract): The goal of this investigation was to identify the Academic Life Experiences that have a major impact upon the Mental Health of young adult students of higher education degrees in the field of the Health Sci-

\footnotetext{
${ }^{1}$ Escola Superior de Saúde da Cruz Vermelha Portuguesa

2 Instituto de Ciências Biomédicas Abel Salazar, Universidade do Porto

A correspondência deve ser enviada para,rnova@esscvp.eu
} 
ences, as well as the domains of Personal Competences that can contribute to the absence of psychological disturbance among them. The research dealt with a sample of 620 people, medical students, nursing students and allied health students: physiotherapy, cardiopulmonary technology and radiographer students, whose average age is 20,3 and ranges between 18 and 25 years old, $81 \%$ being female. The instruments used were a Demographic Questionnaire; The Academic Life Experiences Questionnaire - QVA (Almeida \& Ferreira, 1997, 1999; Ferreira \& Almeida, 1997); The Self-Perception Profile for College Students - SPPCS (Neemann \& Harter, 1986; Pais-Ribeiro 1994) and The Mental Health Inventory - MHI (Pais-Ribeiro, 2001; Veit \& Ware, 1983). Two predictor models of Mental Health were defined for this sample. The first model, which has the Academic Life Experiences as predictors, is composed by the variables Psychological Well-being, Self-confidence, Physical Well-being and Personal Perception of the Cognitive Competences. The second model with the domains of Personal Competences as predictor contains such variables as Global Self-Worth, Intellectual Ability, and Relationships with Parents, Physical Appearance and Romantic Relationships. We discuss the relevance of the final results and their implications, namely the importance of psychological well-being and self-esteem as mental health promoting factors for this population.

Keywords: college students, health sciences, academic life experiences, personal competences, mental health.

\section{Introdução}

A entrada no Ensino Superior corresponde a um período de transição que coloca o jovem perante a necessidade de lidar com diferentes níveis de exigências que, requerendo uma estrutura pessoal consistente, são potencialmente condicionadoras da sua adaptação, podendo comprometer o bem-estar psicológico e a saúde mental.

Os desafios e exigências próprios do contexto do Ensino Superior, que constituem o fundamento das Vivências Académicas (Chickering \& Reisser, 1993; Pascarella \& Terenzini, 2005), podem condicionar o bem-estar psicológico e exigir competências pessoais adequadas e eficazes, que permitam ao jovem manter-se adaptado, de modo a que possa realizar com sucesso as diferentes tarefas (desenvolvimentais e académicas) com as quais tem que se confrontar (Evans, Forney \& Guido-DiBrito, 1998).

A problemática do bem-estar psicológico e da saúde mental dos estudantes do Ensino Superior tem sido bastante abordada na literatura, embora a investigação incida principalmente nos aspectos disfuncionais e de caracterização dos factores de promoção das perturbações mentais e menos nas abordagens da saúde mental positiva e do bem-estar psicológico, conceitos 
que podem ser entendidos como correspondendo a um estado de bem-estar que possibilita aos indivíduos concretizar as suas competências pessoais, lidar eficazmente com os factores de exigência (stress) do seu quotidiano, trabalhar produtivamente e com usufruto pessoal, estando assim aptos para contribuir construtivamente para a sua comunidade de pertença (OMS, 2007).

O stress associado à vida académica surge como tema incontornável na investigação com a população que frequenta o Ensino Superior (McGrath, 2006; Misra, McKean, West \& Russo, 2000; Robotham \& Julian, 2006), nomeadamente no que se refere às suas consequências negativas do ponto de vista emocional e, em particular, na sua relação com o aparecimento de perturbações de ansiedade e de humor (Amada, 2001; Deroma, Leach \& Leverett, 2009; Grayson \& Cooper, 2006; Misra \& McKean, 2000; Nogueira-Martins, Neto, Macedo, Cítero \& Mari, 2004; Rawson, Bloomer \& Kendall, 2001).

Como referem Kadison e DiGeronimo (2004), o stress experimentado pelos estudantes universitários está fortemente relacionado com as vivências académicas, e estes autores afirmam mesmo que os jovens vivem em "crise" no contexto académico, de tal modo que é actualmente comum os alunos apresentarem diferentes tipos de perturbações psicológicas, como: depressão, perturbações do sono, abuso de substâncias, perturbações do comportamento alimentar, comportamentos impulsivos (ex.: promiscuidade sexual e auto-mutilação) e suicídio.

Reconhece-se na literatura especializada (Jeffreys, 2004; Wolf, 2001), que a formação na área das Ciências da Saúde e, em particular, nos cursos de Medicina e Enfermagem, é especialmente exigente no plano psicológico. Estas exigências exercem-se, quer na dimensão cognitiva (ex.: conhecimentos a adquirir que são intensivamente avaliados), quer numa dimensão mais pessoal (ex.: ser capaz de lidar com o sofrimento humano).

Facto consensual para os diferentes autores, são os elevados níveis de stress experimentados pelos alunos do curso de Medicina (Cohen, Rollnick, Smail, Kinnersley, Houston \& Edwards, 2005; Dahlin, Joneborg \& Runeson, 2005; Dyrbye, Thomas \& Shanafelt, 2005; Dyrbye et al., 2006; Morrisson \& Moffat, 2001; Omigbodum, Odukogbe, Omigbodum, Yusuf, Bella \& Olayemi, 2006; Saipanish, 2003; Shaikh et al., 2004; Shaw, Wedding, Zeldow \& Diehl, 2001; Souza \& Menezes, 2005; Stuber, 2006; Tyssen, Vaglum, Grønvold \& Ekeberg, 2001; Tyssen et al., 2007; Wilhelm, 2002; Zonta, Robles \& Grosseman, 2006) e as suas consequências em termos de alterações no bem-estar psicológico e saúde mental, resultando no aparecimento de perturbações mentais, principalmente de cariz ansioso e depressivo (Assadi, Nakhaei, Najafi \& Fazel, 2007; Chandavarkar, Azzam \& Mathews, 2007; Dahlin, Joneborg \& Runeson, 2005; Dyrbye, Thomas \& 
Shanafelt, 2006; Lima, Domingues \& Cerqueira, 2006; Moro, Valle \& Lima, 2005; Rosal, Ockene, Ockene, Barret, Ma \& Hebert, 1997; Rosenthal \& Okie, 2005; Smith, Peterson, Degenhardt \& Johnson, 2007; Yiu, 2005), aumento do consumo de substâncias (Perales, Sogi \& Morales, 2003) e alterações na saúde mental acompanhadas de doença física (Roberts et al., 2001), com consequências em termos de resultados académicos e abandono escolar (Ross, Cleland \& Macleod, 2006).

Alguns estudos têm referido especialmente o primeiro ano do curso de medicina, como sendo o que gera maiores níveis de stress e de morbilidade psicológica (Miller, 1994; Redwood \& Pollack, 2007). Wolf (2001), apresenta como principais stressores no primeiro ano do curso de medicina: estar longe de casa e da família; ter que assimilar muitos conhecimentos; melhorar os seus métodos de estudo; ambiente competitivo de aprendizagem; sentir-se pressionado desde o primeiro dia e deixar de ter tempo para si próprio.

Relativamente aos estudantes de enfermagem, existem autores que referem igualmente elevados níveis de stress relacionados com a vida académica e, paralelamente, risco acrescentado de problemas de saúde mental, também de natureza ansiosa e depressiva (Jones \& Johnston, 1997, 1999, 2000; Lo, 2002; Maville, Kranz \& Tucker, 2004).

No que respeita a outros cursos da área da saúde, não encontrámos na literatura evidência abundante sobre factores relacionados com o stress da vida académica e as suas consequências para os estudantes, embora também estes estudantes apresentem durante a sua formação níveis de stress elevados (Dutta, Pyles \& Miederhoff, 2005; Henning, Ey \& Shaw, 1998), e problemas de saúde mental, como patologia depressiva e uso de substâncias (Stecker, 2004). De referir um estudo comparativo entre estudantes de fisioterapia Australianos e Ingleses, onde se constatam níveis elevados de stress associados ao grau percepcionado das exigências do curso (Tucker, Jones, Mandy \& Gupta, 2006).

Conforme referimos anteriormente, para o confronto com as exigências psicológicas relacionadas com a vida académica, o sujeito deverá possuir determinadas competências pessoais, que permitam atingir níveis de adaptação eficazes e manter o bem-estar psicológico. A avaliação da percepção individual destas competências em estudantes do ensino superior tem sido realizada através da utilização de um modelo multidimensional do auto-conceito (Harter, 1999; Neeman \& Harter, 1986).

Nesta perspectiva, o auto-conceito pode ser definido como a percepção que um indivíduo tem de si próprio nas mais variadas facetas; sejam elas de natureza social, emocional, física ou académica (Hattie, 1992; Harter, 1999). Para Harter (1999), ainda neste âmbito conceptual, a auto-estima ou auto-apreciação global, entendendo-se que a autora considera estes dois 
conceitos como sinónimos (Pais Ribeiro, 2004), depende de dois factores que funcionam de modo aditivo: as competências pessoais e a aprovação social. De acordo com esta perspectiva, as competências em diferentes domínios são importantes para o desenvolvimento da personalidade e concorrem para a auto-estima do indivíduo (Harter, 1989).

Relativamente aos estudantes do Ensino Superior, a relação entre a percepção de competências pessoais, a adaptação académica e o bem-estar psicológico, tem sido abordada em alguns estudos, nomeadamente considerando esta percepção como variável preditora da adaptação académica (Boulter, 2002; Pittman \& Richmond, 2008) ou preditora do bem-estar psicológico (May, Byrd, Brown, Beckman \& Sizemore, 2007; Uhrlass \& Gibb, 2007). Os estudos envolvendo estudantes de Ciências da Saúde têm incidido mais recentemente na relação entre o auto-conceito e a construção da identidade profissional em estudantes de Enfermagem (Holroyd, Bond \& Chan, 2002; Randle, 2006); na percepção de competências académicas de alunos de Medicina (Sobral, 2004) e na auto-estima como preditor de sucesso académico em estudantes de Enfermagem (Peterson, 2009) e ainda, como preditor de bem-estar psicológico em alunos de Enfermagem (Ross, Zeller, Srisaeng, Yimmee, Somchild \& Sawatphanit, 2005; Lo, 2002; Sasat et al., 2002) e de Medicina (Dahlin, Joneborg \& Runeson, 2007).

Face à necessidade de estudos sobre esta problemática na realidade Portuguesa do Ensino Superior na área da Saúde e reconhecendo-se que as exigências da vida académica são factores de stress que podem condicionar a saúde mental, em que as competências pessoais possuídas pelos estudantes se podem reflectir igualmente no seu bem-estar psicológico, são objectivos deste estudo: conhecer quais as vivências académicas que permitem predizer a saúde mental dos estudantes pertencentes a cursos na área das Ciências da Saúde e, quais as competências pessoais que poderão também ter um papel preditor relativamente ao bem-estar psicológico apresentado por estes jovens.

\section{Método}

\section{Participantes}

A amostra deste estudo é constituída por jovens adultos, com idade entre os 18 e os 25 anos, a frequentar, no ano lectivo de 2005/2006, um curso superior na área das ciências da saúde.

Os participantes formam uma amostra intencional, representativa da população em estudo, composta por 620 indivíduos, $80,8 \%$ do sexo feminino, com idades compreendidas entre os 18 e os 25 anos, $M=20,37$ anos, pertencentes a três estabelecimentos de ensino superior, sendo 334 da Esco- 
la Superior de Saúde da Cruz Vermelha Portuguesa, 196 da Escola Superior de Enfermagem da Cruz Vermelha Portuguesa de Oliveira de Azeméis e 90 do Instituto de Ciências Biomédicas de Abel Salazar. Frequentam cursos de Enfermagem $(n=322)$, Fisioterapia $(n=95)$, Medicina $(n=90)$, Cardiopneumologia $(n=75)$ e Radiologia $(n=38)$.

\section{Materiais}

A recolha de dados efectuou-se através de questionários confidenciais de auto-resposta, que incluíam:

Questionário Demográfico, para obtenção de dados respeitantes à caracterização geral dos sujeitos que integraram a amostra.

Questionário de Vivências Académicas (QVA) - (Almeida \& Ferreira, 1997, 1999; Ferreira \& Almeida, 1997). O QVA é um questionário de auto-relato, que avalia, numa perspectiva multidimensional, a forma como os jovens se adaptam a algumas das exigências da vida académica, considerando várias dimensões do ajustamento académico, aprendizagem e desenvolvimento, caracterizadas como promotoras da adaptação ao contexto escolar e contemplando domínios pessoais, relacionais e institucionais. Destina-se a alunos do Ensino Superior Universitário e Politécnico e alguns estudos têm sido realizados em Portugal com estudantes destes níveis de ensino, demonstrando a sua validade (Almeida, Soares \& Ferreira, 1999, 2000; Almeida, Soares, Araújo \& Vila-Chã, 2000; Almeida, Soares, Ferreira \& Tavares, 2004; Araújo, Almeida \& Paúl, 2003; Costa, 2005; Machado \& Almeida, 2000; Machado, Almeida \& Soares, 2002; Santos, 2000). É constituído por 170 itens, distribuídos por 17 subescalas inseridas em três dimensões: Contexto Académico, Aprendizagem e Rendimento Académico e Pessoal e Desenvolvimento Psicossocial. A dimensão Contexto Académico contém as subescalas Adaptação à Instituição (11 itens); Envolvimento em Actividades Extracurriculares (11 itens); Relacionamento com os Colegas (15 itens); Adaptação ao Curso (15 itens); Gestão dos Recursos Económicos (8 itens) e Relacionamento com a Família (10 itens). A dimensão Aprendizagem e Rendimento Académico é composta pelas subescalas Relacionamento com os Professores (14 itens); Métodos de Estudo (11 itens); Bases de Conhecimentos para o Curso (6 itens); Realização de Exames (10 itens); Gestão do Tempo (8 itens). A dimensão Pessoal e Desenvolvimento Psicossocial inclui as subescalas Desenvolvimento de Carreira (14 itens); Autonomia (12 itens); Percepção Pessoal de Competências Cognitivas (10 itens); Auto-Confiança (12 itens); Bem-Estar Psicológico (14 itens) e Bem-Estar Físico (13 iterns). De acordo com Almeida, Ferreira e Soares (2003) o QVA é considerado como instrumento de despiste de dificuldades (screening), e é essencialmente utilizado para fins de investigação, não existindo 
valores normativos para a sua cotação. Importa referir que a pontuação em cada subescala resulta do somatório dos valores em cada item (respondidos através de uma escala de tipo Likert de 1 a 5), correspondendo os valores mais elevados a uma caracterização mais positiva do respectivo domínio (Almeida et al., 2003). No nosso estudo, a consistência interna dos itens das subescalas mostrou o alfa de Cronbach variando entre 0,72 e 0,90.

Mental Health Inventory - MHI (Veit \& Ware, 1983), adaptado à população portuguesa por Pais-Ribeiro (2001). É um questionário de auto-resposta, que tem como objectivo ser uma medida de Saúde Mental na população geral (adultos e adolescentes). Partindo do pressuposto que a Saúde Mental pode ser entendida num continuum, entre uma avaliação de estados positivos e negativos, este instrumento de avaliação psicológica procura detectar, em pessoas que à partida não têm diagnóstico de uma doença mental, alterações no seu estado de saúde, sublinhando a importância do bem-estar subjectivo como um indicador consistente de Saúde Mental (Brook et al., 1979; Ostroff, Woolverton, Berry \& Lesko, 1996; Ware, Johnston, Davies-Avery \& Brook, 1979; Ware, Manning, Duan, Well \& Newhouse, 1984). O MHI é composto por 38 itens que se distribuem pelas subescalas de Ansiedade (10 itens); Depressão, (5 itens); Perda de Controlo Emocional/Comportamental (9 itens); Afecto Positivo (11 itens); Laços Emocionais (3 itens). O Distress Psicológico resulta da soma das subescalas de Ansiedade, Depressão, e Perda de Controlo Emocional/Comportamental; enquanto a de Bem-Estar Psicológico resulta da soma das subescalas Afecto Positivo e Laços Emocionais. É possível calcular um Índice de Saúde Mental (escala MHI completa), que corresponde ao somatório da subescala de Distress Psicológico com o Bem-Estar Psicológico. A resposta a cada item é dada numa escala ordinal (de tipo Likert) com cinco ou seis posições. A nota total resulta da soma dos valores brutos dos itens que compõem cada subescala referida acima. Parte dos itens são cotados de modo invertido. Valores mais elevados correspondem a uma caracterização mais positiva da dimensão em análise. No que respeita à consistência interna, encontrámos neste estudo valores de alfa de Cronbach de 0,97 para a escala completa, variando os valores para as sub-escalas entre 0,73 e 0,96.

Self-Perception Profile for College Students (SPPCS) - (Neemann \& Harter, 1986), adaptado à população portuguesa por Pais-Ribeiro (1994). É uma escala que se propõe avaliar as competências pessoais de estudantes universitários. Este instrumento utiliza para a caracterização do Auto-Conceito de estudantes universitários uma perspectiva multidimensional. A escala, na sua versão original, contém 54 itens (52 na versão portuguesa) distribuídos por 13 subescalas (12 na versão adaptada). O questionário fornece um perfil constituído com as notas das subescalas, em que cada uma se supõe ser uma dimensão relevante para a vida do estudante universitário. 
$\mathrm{Na}$ adaptação do instrumento, existem quatro domínios que se referem a Competências ou Capacidades (Byrne, 1996; Pais-Ribeiro, 1994): Criatividade (4 itens), Competência Académica (5 itens), Competência Intelectual (5 itens) e Competência Atlética (4 itens). Os restantes sete domínios referem-se a Relações Sociais: Aparência (4 itens), Amizades Íntimas (4 itens), Aceitação Social (4 itens), Relações com os Pais (4 itens), Relações Amorosas (4 itens), Humor (3 itens) e Moralidade (4 itens). A subescala de Apreciação Global (7 itens) é independente de domínios, evitando assim uma avaliação do Auto-Conceito Global, resultante da soma dos diferentes domínios. A resposta é dada numa de quatro alternativas: primeiro, pela escolha de uma de duas afirmações antagónicas ("alguns estudantes gostam de ser como são" versus "outros estudantes gostariam de ser diferentes"), sendo a escolhida aquela com a qual o sujeito se identifica. Depois, o sujeito deve centrar-se nessa afirmação escolhendo uma de duas situações: identificação exacta com a afirmação ou apenas aproximação ("sou mesmo assim" ou "sou mais ou menos assim"). Cada item é cotado numa escala de tipo Likert de 1 a 4, indicando a nota mais baixa menor percepção de competência e a mais elevada, melhor percepção de competência. Para cada subescala metade dos itens são formulados pela positiva e a outra metade pela negativa. Os valores obtidos em cada subescala resultam do somatório dos itens que a compõem. A apresentação dos resultados deve efectuar-se através de um perfil, no qual se pode apreciar a percepção que cada sujeito possui face às diferentes competências em avaliação. Na nossa investigação, os valores de consistência interna apresentam o alfa de Cronbach com variação entre 0,65 e 0,87 .

\section{Procedimento}

A recolha de dados decorreu no início do $2 .^{\circ}$ semestre do ano lectivo de 2005/2006. Foi previamente pedida a colaboração de alguns docentes das instituições onde foram recolhidos os dados, os quais disponibilizaram parte do seu horário de leccionação para que o instrumento fosse aplicado aos alunos de cada uma das turmas dos diferentes cursos. Em todos os momentos de aplicação dos questionários foi fornecida informação aos participantes, nomeadamente, sobre os objectivos gerais da investigação, o carácter voluntário da participação (limitada aos alunos com idade entre os 18 e os 25 anos, inclusive) e sobre a confidencialidade dos elementos recolhidos. Nenhum aluno, dos presentes em cada recolha de dados efectuada, se recusou a participar no estudo. Todos os procedimentos estatísticos, relativos ao tratamento dos dados recolhidos, foram efectuados com recurso ao programa SPSS ${ }^{\circledR}$ (Statistical Package for the Social Sciences), versão 15.0 para Windows ${ }^{\circledR}$. 


\section{Resultados}

Com o objectivo de determinar quais as Vivências Académicas que poderiam predizer a Saúde Mental, efectuou-se uma Análise de Regressão Linear Múltipla, usando o método stepwise.

Obteve-se um Modelo de Regressão Linear das Vivências Académicas sobre a Saúde Mental (MHI-Escala Completa/Índice de Saúde Mental), significativo $\left(F_{(4,615)}=340,07, p<0,001\right)$, o qual inclui as subescalas do QVA: Bem-Estar Psicológico, Auto-Confiança, Bem-Estar Físico e Percepção Pessoal de Competências Cognitivas (ver Quadro 1).

Quadro 1. Vivências Académicas preditoras da saúde mental: Modelo de regressão linear

\begin{tabular}{llrrc}
\hline \multicolumn{1}{c}{$\begin{array}{c}\text { Variável } \\
\text { Dependente }\end{array}$} & \multicolumn{1}{c}{ Preditores } & B & \multicolumn{1}{c}{$\boldsymbol{t}$} & \multicolumn{1}{c}{$\boldsymbol{p}$} \\
\hline & Bem-Estar Psicológico & 1,549 & 13,018 & $0,000^{* *}$ \\
& Auto-Confiança & 0,951 & 5,612 & $0,000^{* *}$ \\
Saúde Mental & Bem-Estar Físico & 0,515 & 3,807 & $0,000^{* *}$ \\
$\left(\mathrm{R}^{2} \mathrm{Aj} .=0,69\right)$ & Percepção Pessoal de & 0,457 & 2,328 & $0,020^{*}$ \\
& Competências & & & \\
&
\end{tabular}

Nota: ${ }^{*} p<0,05 ; * * p<0,001$

Este modelo explica 69\% da variância da Saúde Mental na dependência directa dos quatro preditores, permitindo a constituição da seguinte equação de regressão linear: $\mathrm{y}=-1,248+1,549 \mathrm{x}_{1}+0,951 \mathrm{x}_{2}+0,515 \mathrm{x}_{3}+0,457 \mathrm{x}_{4}$, sendo: $\mathrm{y}=$ Saúde Mental; $\mathrm{x}_{1}=$ Bem-Estar Psicológico; $\mathrm{x}_{2}=$ Auto-Confiança; $\mathrm{x}_{3}=$ Bem-Estar Físico e $\mathrm{x}_{4}=$ Percepção Pessoal de Competências Cognitivas.

Com o objectivo de validar o modelo, realizou-se a verificação dos seguintes pressupostos: análise gráfica de resíduos, estudo da multicolinearidade (correlação entre variáveis independentes), análise da homocedasticidade (variância constante dos termos de erro) e medida da auto-correlação. Concluiu-se que a validade preditiva do modelo cumpre todos os pressupostos.

Procurou-se caracterizar as Competências Pessoais que poderiam predizer a Saúde Mental, tendo-se efectuado uma análise de regressão linear múltipla, usando o método stepwise, sendo encontrado um modelo de regressão linear das Competências Pessoais sobre a Saúde Mental (MHI-Escala Completa/Índice de Saúde Mental), significativo $\left(F_{(5,613)}=128,42\right.$, $p<0,001$ ), o qual inclui as subescalas do SPPCS: Apreciação Global, Com- 
petência Intelectual, Relações com os Pais, Aparência e Relações Amorosas (ver Quadro 2).

Quadro 2. Competências Pessoais Preditoras da saúde mental: Modelo de regressão linear.

\begin{tabular}{llcrl}
\hline \multicolumn{1}{c}{$\begin{array}{c}\text { Variável } \\
\text { Dependente }\end{array}$} & \multicolumn{1}{c}{ Preditores } & B & \multicolumn{1}{c}{$\boldsymbol{t}$} & \multicolumn{1}{c}{$\boldsymbol{p}$} \\
\hline & Apreciação Global & 3,221 & 10,990 & $0,000^{* *}$ \\
& Competência Intelectual & 2,246 & 5,839 & $0,000^{* *}$ \\
Saúde Mental & Relações com os Pais & 0,736 & 1,934 & $0,040^{*}$ \\
$\left(\mathrm{R}^{2} \mathrm{Aj} .=0,51\right)$ & Aparência & 0,664 & 2,155 & $0,032^{*}$ \\
& Relações Amorosas & 0,630 & 2,053 & $0,049^{*}$ \\
\hline
\end{tabular}

Nota: ${ }^{*} p<0,05 ; * * p<0,001$

Este modelo explica 51\% da variância da Saúde Mental na dependência directa dos cinco preditores, permitindo a construção da seguinte equação de regressão linear: $y=34,771+3,221 x_{1}+2,246 x_{2}+0,736 x_{3}+0,664 x_{4}+0,630 x_{5}$, sendo: $\mathrm{y}=$ Saúde Mental; $\mathrm{x}_{1}=$ Apreciação Global; $\mathrm{x}_{2}=$ Competência Intelectual; $\mathrm{x}_{3}=$ Relações com os Pais; $\mathrm{x}_{4}=$ Aparência e $\mathrm{x}_{5}=$ Relações Amorosas.

Tal como para o modelo anterior, confirmou-se a validade preditiva através da verificação dos pressupostos de aplicação da regressão linear múltipla, que confirmaram a adequação do modelo.

\section{Discussão}

A análise estatística realizada procurou esclarecer quais as Vivências Académicas e Competências Pessoais que contribuem para a Saúde Mental destes jovens estudantes. Nesse sentido, os dois modelos de regressão linear construídos, permitem alguma clarificação sobre esta questão.

No primeiro modelo as variáveis preditoras pertencem ao domínio pessoal do estudante. A ênfase é colocada no Bem-Estar Psicológico, enquanto medida da satisfação com a vida, estabilidade emocional e afectiva, felicidade e optimismo do estudante; na Auto-Confiança, que depende das expectativas (positivas) pessoais e da inferência das expectativas de colegas e professores, que igualmente deverão ser positivas, sobre as capacidades e desempenho académicos; no Bem-Estar Físico, relacionado principalmente com a ausência de problemas de saúde física e de comportamentos de risco para a saúde e, na Percepção Pessoal de Competências Cognitivas, associa- 
da à auto-eficácia para a resolução de problemas e estruturação do pensamento de modo flexível e profundo.

Esta relação entre Vivências Académicas e Saúde Mental reforça a importância da adaptação pessoal, através da funcionalidade física e mental, face às exigências académicas, não se afastando de contributos empíricos recentes (Turner, Hammond, Gilchrist \& Barlow, 2007; Üner, Özcebe, Telatar \& Tezcan, 2008; Vaez \& Laflamme, 2008), que relacionam, de igual modo, a adaptação académica e a saúde mental, contribuindo para o esclarecimento dos principais factores da vida académica, dos quais depende a saúde mental dos estudantes

O segundo modelo complementa o modelo anterior, sublinhando o contributo da apreciação-global, enquanto medida de auto-estima (Harter, 1999; Mruk, 2006) e das competências de natureza intelectual e social/relacional. Ou seja, a Saúde Mental, surge dependente dos sentimentos e atitudes do jovem face a si mesmo, da crença nas suas capacidades e da importância que considera ter, face aos outros com quem estabelece relações significativas.

A importância da auto-estima para a promoção do bem-estar e da adaptação psicológica surge como um factor consensual na investigação, nomeadamente num entendimento de dimensão geral protectora face ao adoecer mental (Ghaderi, 2006; Harter, Waters \& Whitesell, 1998; Randle, 2003).

Uma apreciação positiva da Competência Intelectual num estudante do ensino superior tem provavelmente um valor acrescentado, pela importância que as capacidades cognitivas têm para o sucesso académico e o não-abandono escolar (Astin, 1993). Por outro lado, confirmam o valor atribuído aos conhecimentos académicos obtidos, os quais são igualmente importantes para as instituições de ensino superior, pois são o seu principal indicador de eficácia (Crosling, Thomas \& Heagney, 2008; Kuh, Kinzie, Schuh \& Whitt, 2005; Yorke \& Longden, 2004).

Também o relevo dado às relações afectivas (familiares e amorosas), sustenta a necessidade sentida pelo jovem de manter proximidade e intimidade, quer com os seus pais, quer no âmbito da vivência da relação amorosa, funcionando ambas como meio de suporte emocional, condição importante para o Bem-Estar Psicológico, por vezes condicionada, principalmente quando o jovem está afastado da família (saída de casa), tem relações familiares perturbadas ou vivencia rupturas amorosas (Diniz, 2005; Hargrove, McDaniel, Malone \& Christiansen, 2006; Whitaker, 2006).

A Aparência, que surge muito associada à auto-imagem e à satisfação com o próprio corpo, é a única dimensão do auto-conceito que pode ser avaliada em todas as etapas do desenvolvimento, podendo igualmente ser entendida como medida de desejabilidade social (Harter, 1999; Mruk, 
2006). Corresponde a uma preocupação mais tradicionalmente associada ao género feminino (não esquecer que a amostra é constituída maioritariamente por jovens do sexo feminino), que em níveis positivos impede o aparecimento de traços psicopatológicos, responsáveis por perturbações depressivas e do comportamento alimentar, comuns nesta fase do desenvolvimento (Harter, 2006).

Em conclusão, podemos caracterizar os modelos obtidos como válidos no sentido do conhecimento sobre a dinâmica da Saúde Mental desta população, aproximando-se de um conceito de Saúde Mental Positiva, alicerçado na funcionalidade do indivíduo, resultado sinérgico do Bem-Estar Psicológico auto-percepcionado e das Competências Pessoais que dinamicamente possibilitam ao sujeito estar activamente adaptado aos seus diferentes contextos de vida.

A valorização destes aspectos deverá, em nosso entender, ser tida em conta no que diz respeito à promoção da saúde mental no contexto do ensino superior na área das ciências da saúde, onde se torna fundamental a criação de estratégias institucionais que permitam o máximo de bem-estar psicológico individual, de modo a que os estudantes possam, nas melhores condições de saúde, enfrentar os exigentes desafios que lhes são colocados pelas diferentes aprendizagens que estes cursos contemplam.

\section{Referências}

Almeida, L. S., \& Ferreira, J. A. (1997). Questionário de vivências académicas (QVA). Braga: Universidade do Minho, Centro de Estudos em Educação e Psicologia.

Almeida, L. S., \& Ferreira, J. A. (1999). Adaptação e rendimento académico no ensino superior: Fundamentação e validação de uma escala de avaliação de vivências académicas. Psicologia: Teoria, Investigação e Prática, 1, 157-170.

Almeida, L. S., Ferreira, J, A., \& Soares, A. P. (2003). Questionário de vivências académicas (Q.V.A. e Q.V.A.-R). In M. M. Gonçalves, M. R. Simões, L. S. Almeida e C. Machado (Coords.), Avaliação psicológica Instrumentos validados para a população portuguesa (Vol. 1, pp. 113-130). Coimbra: Quarteto Editora.

Almeida, L. S., Soares, A. P., \& Ferreira, J. A. (1999). Adaptação, rendimento e desenvolvimento dos estudantes no ensino superior: Construção do questionário de vivências académicas. Braga: Centro de Estudos em Educação e Psicologia, Relatórios de Investigação.

Almeida, L. S., Soares, A. P., \& Ferreira, J. A. (2000). Transição e adaptação à universidade: Apresentação de um questionário de vivências académicas (QVA). Psicologia, XIV (2), 189-208. 
Almeida, L. S., Soares, A. P., Araújo, S., \& Vila-Chã, M. C. (2000). Questionário de vivências académicas: Precisão das subescalas. In A. Soares, A. Osório, J. V. Capela, 1. S. Almeida, R. M. Vasconcelos \& S. M. Caires (Eds.), Transição para o ensino superior (pp. 81-90). Braga: Universidade do Minho, Conselho Académico.

Almeida, L. S., Soares, A. P., Ferreira, J, A., \& Tavares, J. (2004). Transição e adaptação à universidade: Apresentação de alguns instrumentos de medida. In C. Machado, L. S. Almeida, M. Gonçalves \& V. Ramalho (Orgs.), Avaliação psicológica: Formas e contextos (pp. 487-495). Braga: Psiquilíbrios Edições.

Amada, G. (2001). Mental health and student conduct issues on the college campus: $A$ reading. Asheville, NC: College Administration Publications.

Araújo, B. R., Almeida, L. R., \& Paúl, M. C. (2003). Transição e adaptação académica dos estudantes à escola de enfermagem. Revista Portuguesa de Psicossomática, 5 (1), 56-64.

Assadi, S. M., Nakhaei, M. R., Najafi, F., \& Fazel, S. (2007). Mental health in three generations of Iranian medical students and doctors. Social Psychiatry Psychiatric Epidemiology, 42, 57-60.

Astin, A. W. (1993). What matters in college? Four critical years revisited. San Francisco: Jossey-Bass.

Boulter, L. T. (2002). Self-concept as a predictor of college freshman academic adjustment. College Student Journal, 36 (2), 234-246.

Brook, R. H., Ware, J. E., Davies-Avery, A., Stewart, A. L., Donald, C. A., Rogers, W. H., Williams, K. N., \& Johnston, S. A. (1979). Overview of adults health status measures fielded in Rand's health insurance study. Medical Care, 17 (7) (supplement).

Byrne, B. M. (1996). Measuring self-concept across the life span: Issues and instrumentation. Washington, DC: American Psychological Association.

Chandavarkar, U., Azzam, A., \& Mathews, C. A. (2007). Anxiety symptoms and perceived performance in medical students. Depression and Anxiety, 24, 103-111 .

Chickering, A. W., \& Reisser, L. (1993). Education and identity. San Francisco: Jossey-Bass.

Cohen, D., Rollnick, S., Smail, S., Kinnersley, P., Houston, H., \& Edwards, K. (2005). Communication, stress and distress: Evolution of an individual support programme for medical students and doctors. Medical Education, 28, 3-4.

Costa, E. S. (2005). Um olhar sobre a saúde dos estudantes do ensino superior. Lisboa: Instituto Piaget.

Crosling, G., Thomas, L., \& Heagney, M. (2008). Introduction: Student success and retention. In G. Crosling, L. Thomas \& M. Heagney (Eds.), Improving students retention in higher education: The role of teaching and learning (pp. 1-13). Abingdon: Routledge.

Dahlin, M., Joneborg, N., \& Runeson, B. (2005). Stress and depression among medical students: a cross-sectional study. Medical Education, 39, 594-604. 
Dahlin, M., Joneborg, N., \& Runeson, B. (2007). Performance-based self-esteem and burnout in a cross-sectional study of medical students. Medical Teacher, $29,43-48$.

Deroma, V. M., Leach, J. B., \& Leverett, J. P. (2009). The relationship between depression and college students. College Student Journal, 43 (2), 325-334.

Diniz, A. M. (2005). A universidade e os seus estudantes: Um enfoque psicológico. Lisboa: Instituto Superior de Psicologia Aplicada.

Dutta, A. P., Pyles, M. A., \& Miederhoff, P. A. (2005). Stress in health professions students: Myth or reality? A review of the existing literature. Journal of $\mathrm{Na}$ tional Black Nurses Association, 16 (1), 63-68.

Dyrbye, L. N., Thomas, M. R., \& Shanafelt, T. D. (2005). Medical student distress: Causes, consequences, and proposed solutions. Mayo Clinic Proceedings, 80, 1613-1622.

Dyrbye, L. N., Thomas, M. R., \& Shanafelt, T. D. (2006). Systematic review of depression, anxiety, and other indicators of psychological distress among US and Canadian medical students. Academic Medicine, 81(4), 354-373.

Dyrbye, L. N., Thomas, M. R., Huntington, J. L., Lawson, K. L., Novotny, P. J., Sloan, J. A., \& Shanafelt, T. D. (2006). Personal life events and medical student burnout: A multicenter study. Academic Medicine, 81 (4), 374-384.

Evans, N. J., Forney, D. S., \& Guido-Dibrito, F. (1998). Student development in college: Theory, research and practice. San Francisco: Jossey-Bass.

Ferreira, J. A., \& Almeida, J. S. (1997). Questionário de vivências académicas (QVA): Fundamentação e procedimentos preliminares de construção. In M. Gonçalves, I. Ribeiro, S. Araújo, C. Machado, L. Almeida \& M. Simões (Orgs.), Avaliação psicológica: Formas e contextos (pp. 441-452). Braga: APPORT.

Ghaderi, A. (2006). The foundation of the self and the assessment of self-esteem. In A. P. Prescott (Ed.), The concept of self in psychology (pp. 1-19). New York: Nova Science Publishers.

Grayson, P. A., \& Cooper, S. (2006). Depression and anxiety. In P. A. Grayson \& P. W. Meilman (Eds.). College mental health practice (pp. 113-134). New York: Routledge.

Hattie, J. (1992). Self-concept. Hillsdale, NJ: Lawrence Eribaum Associates.

Hargrove, D. S., McDaniel, S. H., Malone, E., \& Christiansen, M. H. (2006). Family problems. In P. A. Grayson \& P. W. Meilman (Eds.), College mental health practice (pp. 79-93). New York: Routledge.

Harter, S. (1989). Causes, correlates, and the funcional role of global sef-worth: A life-span perspective. In J. Kolligian \& R. Sternberg (Eds.), Perceptions of competence and incompetense across the life-span. New Haven: Yale University Press.

Harter, S. (1999). The construction of the self: A developmental perspective. New York: Guilford Press.

Harter, S. (2006). Self-processes and developmental psychopathology. In D. Cicchetti \& D. J. Cohen (Eds.), Developmental psychopathology $\left(2^{\text {nd }}\right.$ ed.) (Vol. 1, pp. 370-418). Hoboken, NJ: John Wiley \& Sons. 
Harter, S., Waters, P., \& Whitesell, N. (1998). Relational self-worth: Differences in perceived worth as a person across interpersonal contexts among adolescents. Child Development, 69 (3),756-766.

Henning, K., Ey, S., \& Shaw, D. (1998). Perfectionism, the impostor phenomenon and psychological adjustment in medical, dental, nursing and pharmacy students. Medical Education, 32, 456-464.

Holroyd, E. A., Bond, M. H., \& Chan, H. Y. (2002). Perceptions of sex-role stereotypes, self-concept, and nursing role ideal in Chinese nursing students. Journal of Advanced Nursing, 37 (3), 294-303.

Jeffreys, M. R. (2004). Nursing student retention: Understanding the process and making a difference. New York: Springer Publishing Company.

Jones, M. C., \& Johnston, D. W. (1997). Distress, stress and coping in first-year student nurses. Journal of Advanced Nursing, 26, 475-482.

Jones, M. C., \& Johnston, D. W. (1999). The derivation of a brief student nurse stress index. Work \& Stress, 13 (2), 162-181.

Jones, M. C., \& Johnston, D. W. (2000). Reducing distress, in first level and student nurses: A review of the applied stress management literature. Journal of $\mathrm{Ad}$ vanced Nursing, 32 (1), 66-74.

Kadison, R., \& DiGeronimo, T. F. (2004). College of the overwhelmed: The campus mental health crisis and what to do about it. San Francisco: Jossey-Bass.

Kuh, G. D., Kinzie, J., Schuh, J. H., \& Whitt, E. J. (2005). Student success in college: Creating conditions that matter. San Francisco: Jossey-Bass.

Lima, M. C., Domingues, M. S., \& Cerqueira, A. T. (2006). Prevalência e fatores de risco para transtornos mentais comuns entre estudantes de medicina. Revista de Saúde Pública, 40 (6), 1035-1041.

Lo, R. (2002). A longitudinal study of perceived level of stress, coping and self-esteem of undergraduate nursing students: An Australian case study. Journal of Advanced Nursing, 39 (2), 119-126.

Machado, C., \& Almeida, L. S. (2000). Vivências académicas: Análise diferencial em estudantes dos $1 .^{\circ}$ e $4 .^{\circ}$ anos do ensino superior. In J. Tavares \& R. A. Santiago (Orgs.), Ensino superior: (In)sucesso académico (pp. 133-145). Porto: Porto Editora.

Machado, C., Almeida, L. S., \& Soares, A. P. (2002). Academic experience at the beginning and the end of university studies. European Journal of Education, 37 (4), 387-394.

Maville, J. A., Kranz, P. L., \& Tucker, B. A. (2004). Perceived stress reported by nurse practitioner students. Journal of the American Academy of Nurses Practitioners, 16 (6), 257-262.

May, L. N., Byrd, D. A., Brown, K., Beckman, T., \& Sizemore, J. (2007). Exploring the relationships among hurt reactivity, social adjustment and psychological well-being. Psychology Journal, 4 (2), 50-57.

McGrath, R. (2006). Stress. In P. A. Grayson \& P. W. Meilman (Eds.), College mental health practice (pp. 135-151). New York: Routledge.

Miller, P. M. (1994). The first year at medical school: Some findings and student perceptions. Medical Education, 28, 5-7. 
Misra, R., \& McKean, M. (2000). College students' academic stress and its relation to their anxiety, time management, and leisure satisfaction. American Journal of Health Studies, 16 (1), 41-51.

Misra, R., McKean, M., West, S., \& Russo, T. (2000). Academic stress of college students: Comparison of student and faculty perceptions. College Student Journal, 34 (2), 236-245.

Moro, A., Valle, J. B., \& Lima, L. P. (2005). Sintomas depressivos nos estudantes de medicina da universidade da região de Joinville (SC). Revista Brasileira de Educação Médica, 29 (2), 97-102.

Morrison, J., \& Moffat, K. (2001). More on medical students stress. Medical Education, 35, 617-618.

Mruk, C. J. (2006). Self-esteem research, theory and practice: Toward a positive psychology of self-esteem ( $3^{\text {rd }}$ ed.). New York: Springer Publishing Company.

Neemann, J., \& Harter, S. (1986). Manual for the self-perception profile for college students. Denver: University of Denver.

Nogueira-Martins, L. A., Neto, R. F., Macedo, P. C., Cítero, V. A., \& Mari, J. J. (2004). The mental health of graduate students at the federal University of São Paulo: A preliminary report. Brazilian Journal of Medical and Biological Research, 37, 1519-1524.

Omigbodun, O., Odukogbe, A.-T., Omigbodun, A., Yusuf, O., Bella, T., \& Olayemi, O. (2006). Stressors and psychological symptoms in students of medicine and allied health professions in Nigeria. Social Psychiatry Psychiatric Epidemiology, 41, 415-421.

OMS (2007). Mental health: Strengthening mental health promotion. Fact sheet n. ${ }^{\circ} 220$. Geneva: World Health Organization.

Ostroff, J. S., Woolverton, K. S., Berry, C., \& Lesko, L. M. (1996). Use of the mental health inventory with adolescents: A secondary analysis of the rand health insurance study. Psychological Assessment, 8 (1), 105-107.

Pais-Ribeiro, J. L. (1994). Adaptação do self-perception profile for college students à população portuguesa: Sua utilização no contexto da psicologia da saúde. In L. Almeida \& I. Ribeiro (Eds.), Avaliação psicológica: Formas e contextos (pp. 129-138). Braga: APPORT.

Pais-Ribeiro, J. L. (2001). Mental health inventory: Um estudo de adaptação à população portuguesa. Psicologia, Saúde e Doenças, 2 (1), 77-99.

Pais Ribeiro, J. (2004). Desenvolvimento de uma escala de auto-apreciação pessoal ou auto estima para utilização em contexto de saúde. Psicologia e Educação, 3 (2), 59-66.

Pascarella, E. T., \& Terenzini, P. T. (2005). How college affects students: A third decade of research. San Francisco: Jossey-Bass.

Perales, A., Sogi, C., \& Morales, R. (2003). Estúdio comparativo de salud mental en estudiantes de medicina de dos universidades estatales Peruanas. Anales de la Faculdad de Medicina, 64 (4), 239-246.

Peterson, V. M. (2009). Predictors of academic success in first semester baccalaureate nursing students. Social Behavior and Personality, 37 (3), 411-418. 
Pittman, L. D., \& Richmond, A. (2008). University belonging, friendship quality, and psychological adjustment during the transition to college. The Journal of Experimental Education, 76 (4), 343-361.

Randle, J. (2003). Bullying in the nursing profession. Journal of Advanced Nursing, 43 (4), 395-401.

Randle, J. (2006). Psychology of the self. In A. P. Prescott (Ed.), The concept of self in medicine and health care (pp. 75-96). New York: Nova Publisher.

Rawson, H. E., Bloomer, K., \& Kendall, A. (2001). Stress, anxiety, depression, and physical illness in college students. Journal of Genetic Psychology, 155 (3), 321-330.

Redwood, S. K., \& Pollak, M. H. (2007). Student-led stress management program for first-year medical students. Teaching and Learning in Medicine, 19, 42-46.

Roberts, L. W., Warner, T. D., Lyketsos, C., Frank, E., Ganzini, L., Carter, D., \& Collaborative Research Group on Medical Student Health (2001). Perceptions of academic vulnerability associated with personal illness: A study of 1,027 students at nine medical schools. Comprehensive Psychiatry, 42 (1), 1-15.

Robotham, D., \& Julian, C. (2006). Stress and the higher education student: A critical review of the literature. Journal of Further and Higher Education, 30 (2), 107-117.

Rosal, M. C., Ockene, I. S., Ockene, J. K., Barrett, S. V., Ma, Y., \& Hebert, J. R. (1997). A longitudinal study of students' depression at one medical school. Academic Medicine, 72 (6), 542-546.

Rosenthal, J. M., \& Okie, S. (2005). White coat, mood indigo - depression in medical school. The New England Journal of Medicine, 353 (11), 1085-1088.

Ross, R., Zeller, R., Srisaeng, P., Yimmee, S., Somehid, S., \& Sawatphanit, S. (2005). Depression, stress, emotional support, and self-esteem among baccalaureate nursing students in Thailand. International Journal of Nursing Education Scholarship, 2 (1), 0-15.

Ross, S., Cleland, J., \& Macleod, M. J. (2006). Stress, debt and undergraduate medical student performance. Medical Education, 40, 584-589.

Saipanish, R. (2003). Stress among medical students in a Thai medical school. Medical Teacher, 25 (5), 502-506.

Santos, L. (2000). Vivências académicas e rendimento escolar: Estudo com alunos universitários do $1 .^{\circ}$ ano. Dissertação de Mestrado em Psicologia Escolar apresentada ao Instituto de Educação e Psicologia da Universidade do Minho, Braga.

Sasat, S., Burnard, P., Edwards, D., Naiyapatana, W., Hebden, U., Boonrod, W., Arayathanitkul \& Wongmak, W. (2002). Self-esteem and student nurses: A cross-cultural study of nursing students in Thailand and the UK. Nursing and Health Sciences, 4, 9-14.

Shaikh, B. T., Kahloon, A., Kazmi, M., Khalid, H., Nawaz, K., Khan, N. A., \& Khan, S. (2004). Students, stress and coping strategies: A case of Pakistani medical school. Education for Health, 17 (3), 346-353. 
Shaw, D. L., Wedding, D., Zeldow, P. B., \& Diehl, N. (2001). Special problems of medical students. In D. Wedding (Ed.), Behavior and medicine ( $3^{\text {th }}$ ed., rev.) (pp. 67-84). Seattle, WA: Hogrefe \& Huber.

Smith, C. K., Peterson, D. F., Degenhardt, B. F., \& Johnson, J. C. (2007). Depression, anxiety, and perceived hassles among entering medical students. Psychology, Health \& Medicine, 12 (1), 31-39.

Sobral, D. J. (2004). Medical students' self-appraisal of first-year learning outcomes: use of the course valuing inventory. Medical Teacher, 26 (3), 234-238.

Souza, F. G., \& Menezes, M. G. (2005). Estresse nos estudantes de medicina da universidade Federal do Ceará. Revista Brasileira de Educação Médica, 29 (2), 91-96.

Stecker, T. (2004). Well-being in an academic environment. Medical Education, 38, 465-478.

Stuber, M. L. (2006). Medical student and physician well-being. In D. Wedding \& M. L. Stuber (Eds.), Behavior \& medicine (4 ${ }^{\text {th }}$ ed., rev.) (pp. 167-174). Cambridge, MA: Hogrefe \& Huber.

Tucker, B., Jones, S., Mandy, A., \& Gupta, R. (2006). Physiotherapy students' sources of stress, perceived course difficulty, and paid employment: Comparison between Western Australia and United Kingdom. Physiotherapy Theory and Practice, 22 (6), 317-328.

Turner, A. P., Hammond, C. L., Gilchrist, M., \& Barlow, J. H. (2007). Coventry university students' experience of mental health problems. Counselling Psychology Quarterly, 20 (3), 247-252.

Tyssen, R., Vaglum, P., Grønvold, N. T., \& Ekeberg, Ø. (2001). Factors in medical school that predict postgraduate mental health problems in need of treatment: A nationwide and longitudinal study. Medical Education, 35, 110-120.

Tyssen, R., Dolatowski, F. C., Røvik, J. O., Thorkildsen, R. F., Ekeberg, Ø., Hem, E., Gude, T., Grønvold, N. T., \& Vaglum, P., (2007). Personality traits and types predict medical school stress: A six-year longitudinal and nationwide study. Medical Education, 41(8), 781-787.

Uhrlass, D. J., \& Gibb, B. E. (2007). Negative life events, self-perceived competence, and depressive symptoms in young adults. Cognitive Therapy and Research, 31 (6), 773-783.

Üner, S., Özcebe, H., Telatar, T. G., \& Tezcan, S. (2008). Assessment of mental health of university students with GHQ-12. Turkish Journal of Medical Sciences, 38 (5), 437-446.

Vaez, M., \& Laflamme, L. (2008). Experienced stress, psychological symptoms, self-rated health and academic achievement: A longitudinal study of Swedish university students. Social Behavior and Personality, 36 (2), 183-196.

Veit, C., \& Ware, J. (1983). The structure of psychological distress and well-being in general populations. Journal of Consulting and Clinical Psychology, 51 (5), 730-742.

Ware, J. E., Johnston, S. A., Davies-Avery, A., \& Brook, R. H. (1979). Conceptualization and measurement of health for adults in the health insurance study: Vol. III. Mental Health (R-1987/3-HEW). Santa Monica, CA: Rand Corporation. 
Ware, J. E., Manning, W. G., Duan, N., Well, K. B., \& Newhouse, J. P. (1984). Health status and the use of outpatient mental health services. American Psychologist, 39 (10), 1090-1100.

Whitaker, L. C. (2006). Relationships. In P. A. Grayson \& P. W. Meilman (Eds.), College mental health practice (pp. 95-112). New York: Routledge.

Wilhelm, K. A. (2002). The student and junior doctor in distress. Medical Journal of Australia, 177 (1 Suppl.), S5-S8.

Wolf, T. M. (2001). To your health: Achieving well-being during medical school. Thousand Oaks, CA: Sage Publications.

Yiu, V. (2005). Supporting the well-being of medical students. Canadian Medical Association Journal, 172 (7), 889-890.

Yorke, M., \& Longden, B. (2004). Retention and student success in higher education. Berkshire: Society for Research into Higher Education \& Open University Press.

Zonta, R., Robles, A. C., \& Grossman, S. (2006). Estratégias de enfrentamento do estresse desenvolvidas por estudantes de medicina da Universidade Federal de Santa Catarina. Revista Brasileira de Educação Médica, 30 (3),147-153. 